\title{
Immunoexpression of DNA fragmentation factor 40, DNA fragmentation factor 45, and B-cell lymphoma 2 protein in normal human endometrium and uterine myometrium depends on menstrual cycle phase and menopausal status
}

\author{
Tomasz Banas ${ }^{1}$, Kazimierz Pitynski ${ }^{1}$, Krzysztof Okon², Marcin Mikos ${ }^{3}$, Aleksandra I. Czerw ${ }^{4}$, \\ Andrzej Deptała ${ }^{5}$, Artur Ludwin ${ }^{1}$
}

\author{
${ }^{1}$ Department of Gynecology and Oncology, Jagiellonian University Medical College, \\ Krakow, Poland \\ 2Department of Pathomorphology, Jagiellonian University Medical College, Krakow, \\ Poland \\ ${ }^{3}$ Dietl Specialist Hospital, Krakow, Poland \\ ${ }^{4}$ Department of Public Health, Medical University of Warsaw, Warsaw, Poland \\ ${ }^{5}$ Department of Cancer Prevention, Medical University of Warsaw, Warsaw, Poland
}

Submitted: 13 June 2017

Accepted: 7 July 2017

Arch Med Sci 2018; 14, 6: 1254-1262

DOI: https://doi.org/10.5114/aoms.2017.69383

Copyright @ 2017 Termedia \& Banach

\section{Abstract}

Introduction: DNA fragmentation factors 40 and 45 (DFF40 and DFF45) are final executors of apoptosis, and B-cell lymphoma $2(\mathrm{Bcl}-2)$ is a well-recognized apoptosis inhibitor. We aimed to evaluate DFF40, DFF45 and $\mathrm{Bcl}-2$ immunoexpression in the normal human endometrium with respect to the glandular and stromal layer and in uterine myometrium.

Material and methods: DFF40, DFF45, and $\mathrm{Bcl}-2$ expression was assessed via immunohistochemistry in the endometrium and myometrium collected postmenopausally and premenopausally during the proliferative and secretory phases of the menstrual cycle.

Results: Compared to the myometrium and stroma, endometrial glands showed the highest DFF40 and DFF45 expression in pre- and postmenopausal specimens. DFF45, but not DFF40, glandular expression dependent on menstrual cycle phase and DFF40 and DFF45 scoring was significantly lower in postmenopausal specimens. Significantly higher Bcl-2 expression was observed in proliferative glandular endometrium compared to secretory and postmenopausal specimens. No cycle- or menopause-dependent changes were reported for stromal or myometrial DFF40, DFF45 or Bcl-2 expression. DFF40, DFF45 and Bcl-2 expression was independent of age, age at menarche and menopause, $\mathrm{BMI}$, menstrual cycle and menses lengths, parity and gravidity.

Conclusions: The study provides important evidence regarding menstrual cycle-dependent changes in the expression of DFF40, DFF45 and Bcl-2 in the normal human endometrium, especially in the glandular layer, and shows that their levels are stable in the normal uterine myometrium.

Key words: endometrium, $\mathrm{Bcl}-2$, menstrual cycle, DNA fragmentation factors 40, DFF40, DNA fragmentation factor 45, DFF45, B-cell lymphoma 2, uterine myometrium.

\section{Corresponding author:}

Aleksandra I. Czerw PhD

Department of Public Health

Medical University

of Warsaw

61 Zwirki Wigury St

08-456 Warsaw, Poland

Phone: +48 501176370

E-mail: ola_czerw@wp.pl 


\section{Introduction}

During the reproductive period, the human non-pregnant uterus undergoes hormonal-dependent cyclic changes, and menstruation is the result of enhanced apoptosis [1]. Abnormal uterine bleeding, although non-pathognomonic, may be the first manifestation of endometrial pathology including malignancies [2]. In our previous studies we observed increased expression of apoptosis-related DNA fragmentation factor 45 (DFF45) in the endometrial glands of the secretory endometrium; unfortunately, we did not investigate myometrial expression of DFF45 and other apoptosis-related factors at that time [3, 4].

DFF45 acts as a chaperone of DNA fragmentation factor 40 (DFF40) and serves as a substrate for caspase-3 $[5,6]$. The activation of the apoptotic cascade results in DF45 cleavage via the activated caspase-3, causing DFF40 release and oligonucleosomal DNA degradation [7]. According to Widlak et al., DFF45 is not a simple DFF40 inhibitor; rather, it acts as a DFF40 chaperone that is responsible for the proper folding of DFF40 to acquire its biological function [7]. Abundant DFF40 and DFF45 expression has been observed in ovarian endometriomas, endometrial cancer and other malignancies such as ovarian epithelial cancers, colon and esophageal cancers, as well as glioblastoma [8-13].

In contrast to the DFF40/DFF45 complex, the $\mathrm{Bcl}-2$ protein is a core mitochondrial anti-apoptotic factor that has been widely investigated with regard to the human reproductive tract, including benign endometrial and myometrial disorders, as well as malignancies [14-16]. Decreased Bcl-2 was confirmed in the human secretory endometrium, ovarian endometriosis, human stromal cell lines (ThESC, ATCC, 4003) treated with raloxifene and also in the lamina propria lymphocytes of intestinal tissues from patients with Crohn's disease [16-18]. The Bcl-2 protein, which is located predominantly in the mitochondrial inner membrane, blocks the recruitment of proapoptotic factors such as Bax and stabilizes the mitochondrial membranes, thereby blunting the intrinsic death signaling pathway [19]. Additionally, together with $\mathrm{BCl}-\mathrm{XL}, \mathrm{BCl}-2$ is responsible for preventing cytochrome c from triggering caspase-9 activity [17].

The present study evaluated DFF40, DFF45, and $\mathrm{Bcl}-2$ expression in the human physiological endometrium and myometrium with respect to menstrual cycle phases and menopausal status because the results may assist in the interpretation of their expression in pathological findings.

\section{Material and methods}

\section{Case selection}

This retrospective study of paraffin-embedded slides was approved by the Jagiellonian Univer- sity Review Board. The endometrial specimens were collected during hysteroscopic procedures in patients with an initial diagnosis of uterine malformation $(n=21)$, endometrial polyps $(n=47)$ or submucosal myoma $(n=9)$ that finally were excluded based on their hysteroscopy and histopathological results. Myometrial samples were acquired from women with persistent cervical intraepithelial neoplasia (CIN) (19 cases of CIN2 and 17 cases of CIN3) observed over a 12-month follow-up after cervical conisation) or recurrent (21 cases of CIN2 and 8 cases of CIN3) premalignant cervical pathology, who were qualified for total hysterectomy as a final treatment. The pathology of the uterine corpus was not observed in any of the cases and each patient contributed only one specimen. Patients who: (1) had a history of malignancy; (2) smoked; (3) suffered from polycystic ovarian syndrome; and (4) were prescribed hormonal treatment within the past 5 years were not eligible for this study. 'Menopause' was defined as the date of the final menstrual period, with no menses reported during the subsequent 12-month period. Menstrual cycle characteristics were based on patient self-reports.

\section{Immunohistochemistry and immunohistochemical scoring}

From each case, a representative tissue block was selected, deparaffinized and rehydrated as described previously [3, 4, 8]. A standard immunohistochemical technique was performed using a rabbit polyclonal antibody to DFF45 (Abcam, Cambridge, UK) at a dilution of $1: 100$, a rabbit polyclonal antibody to DFF40 (Abcam, Cambridge, UK) at a dilution of $1: 50$, and a monoclonal mouse anti-human antibody to Bcl-2 (Leica Microsystems $\mathrm{GmbH}$, Wetzlar, Germany) at a dilution of 1 : 200. Slides were incubated with 3,3'-diaminobenzidine for $5 \mathrm{~min}$ and counterstained with hematoxylin for $30 \mathrm{~s}$; the enzymatic reactivity was visualized. A colon carcinoma sample for $\mathrm{Bcl}-2$, a human breast carcinoma tissue for DFF45, and human ovary tissue sections for DFF40 were used as positive controls. For the negative control, the same specimens and methods were used, but the primary antibodies were omitted.

Two board-certified histopathologists blindly evaluated the DFF45, DFF40, and Bcl-2 staining for each slide using 5 high-power fields (40x) of maximal staining intensity. Each tissue was scored based on the intensity of staining ( 0 , no staining; 1 , weak staining; 2 , moderate staining; and 3 , strong staining) and the number of stained cells $(0$, expression in up to $10 \%$ of the cells; $1+$, expression in $10-50 \%$ of the cells; $2+$, expression in $51-80 \%$ of the cells; and $3+$, expression in more than $80 \%$ of the cells). The final immunoreactivity 
score was determined by multiplying the intensity scores by the extent of the positivity scores of the stained cells to provide a score that ranged from 0 to 12 . A discrepancy between the observations occurred in 18 (2.74\%) cases, and the samples were verified again to achieve a consensus. Therefore, K.O. and H.M-O. performed another evaluation of selected slides 2 weeks after the primary evaluation to prevent recall bias.

\section{Statistical analysis}

The clinical features of the study groups were compared using one-way analysis of variance (ANOVA) or the Kruskal-Wallis test, depending on the homogeneity of variance, while differences in the immunohistochemical scoring were evaluated using the Kruskal-Wallis test. Post hoc tests were used where appropriate. The non-parametric Wilcoxon paired test was used and the Mann-Whitney $U$ test was applied to compare scorings between endometrial gland stroma and myometrium. Clinical features were shown as the mean \pm standard deviation (SD). Data from the immunohistochemistry results were presented as the median and interquartile range (IQR). A multiple step-wise regression was used to evaluate the associations between DFF40, DFF45, and Bcl-2 expression and clinical characteristics. To evaluate the intra-rater agreement of immunohistochemistry scoring, kappa statistics with a $p$-value were applied. To randomize the patients who underwent evaluation for the intra-rater agreement, we used the research randomizer (www.randomizer.org), and 30 (20.40\%) samples were randomly, separately, and distinctly chosen from a total of 142 samples regarding DFF40, DFF45, and $\mathrm{Bcl}-2$ for observer 1 and observer 2. The Guidelines for Reporting Reliability and Agreement in Studies were used to verify these results [20]. A p-value of less than 0.05 was considered significant. All calculations were performed using Statistica version 12.0 (StatSoft, Inc. 2014. Statistica, version 12; www.statsoft.com).

\section{Results}

\section{Patients and materials}

The tissue samples included proliferative $(n=25)$, secretory $(n=25)$, and atrophic $(n=27)$ endometrium and myometrium collected during the proliferative $(n=24)$ and secretory $(n=20)$ phases of the menstrual cycle as well as postmenopausally $(n=21)$. Table I presents clinical characteristics of donors.

\section{DFF40 and DFF45 expression}

DFF40 and DFF45 nuclear expression was observed in endometrium and myometrium (Figure 1).
Median DFF40 expression in proliferatory and secretory glandular endometrium was significantly higher compared to glandular atrophic endometrium, whereas no such changes were observed in stromal endometrium and myometrium (Figure 2, Table II). The significantly highest median DFF40 scoring was observed in glandular endometrium compared the endometrial stroma and myometrium in pre- and postmenopausal specimens (Table II).

The median DFF45 expression in the endometrial glandular epithelium was significantly higher during the secretory phase of the menstrual cycle than during the proliferative phase and when compared with postmenopausal samples (Table II, Figure 2). No differences in DFF45 scoring were observed in endometrial stroma or myometrium with respect to menstrual cycle phases or menopausal status (Table II, Figure 2). Significantly higher median DFF45 expression was proved in endometrial glands compared to the stroma and myometrium with respect to phases of the menstrual cycle and menopausal status (Table II).

\section{Bcl-2 expression}

Bcl-2 presented cytoplasmic expression with the highest median scoring observed with regard to the glandular layer of the proliferative endometrium, and it differed significantly from the median expression observed in the endometrial stroma and myometrium derived during the proliferative phase of the menstrual cycle (Figure 1, Table II). No differences in the median $\mathrm{Bcl}-2$ expression were found among the endometrial glandular epithelium, endometrial stroma, or uterine myometrium in the postmenopausal samples (Table II). Subsequently, only the expression of $\mathrm{Bcl}-2$ in the endometrial glandular epithelium was dependent on the menstrual cycle phases, showing the highest median expression during the proliferative phase (Figure 2, Table II).

\section{Association of clinical features and DFF40, DFF45, and Bcl-2 endometrial and myometrial expression}

The expression of DFF40, DFF45 and $\mathrm{Bcl}-2$ in endometrial glands and stroma shows no association with age, age at menarche, body mass index (BMI), length of menstrual cycle and menses, parity or gravidity, while the presence of the menopause was associated negatively with DFF40 and DFF45 expression in endometrial glands ( $p=0.006 ; p=0.005$, respectively), and DFF45 glandular expression alone was dependent on the phase of the menstrual cycle $(p=0.002)$. Additionally, $\mathrm{Bcl}-2$ glandular expression was dependent on the menstrual cycle phase $(p<0.001)$.

Myometrial DFF40, DFF45 and Bcl-2 expression was independent from all the above clinical features. 


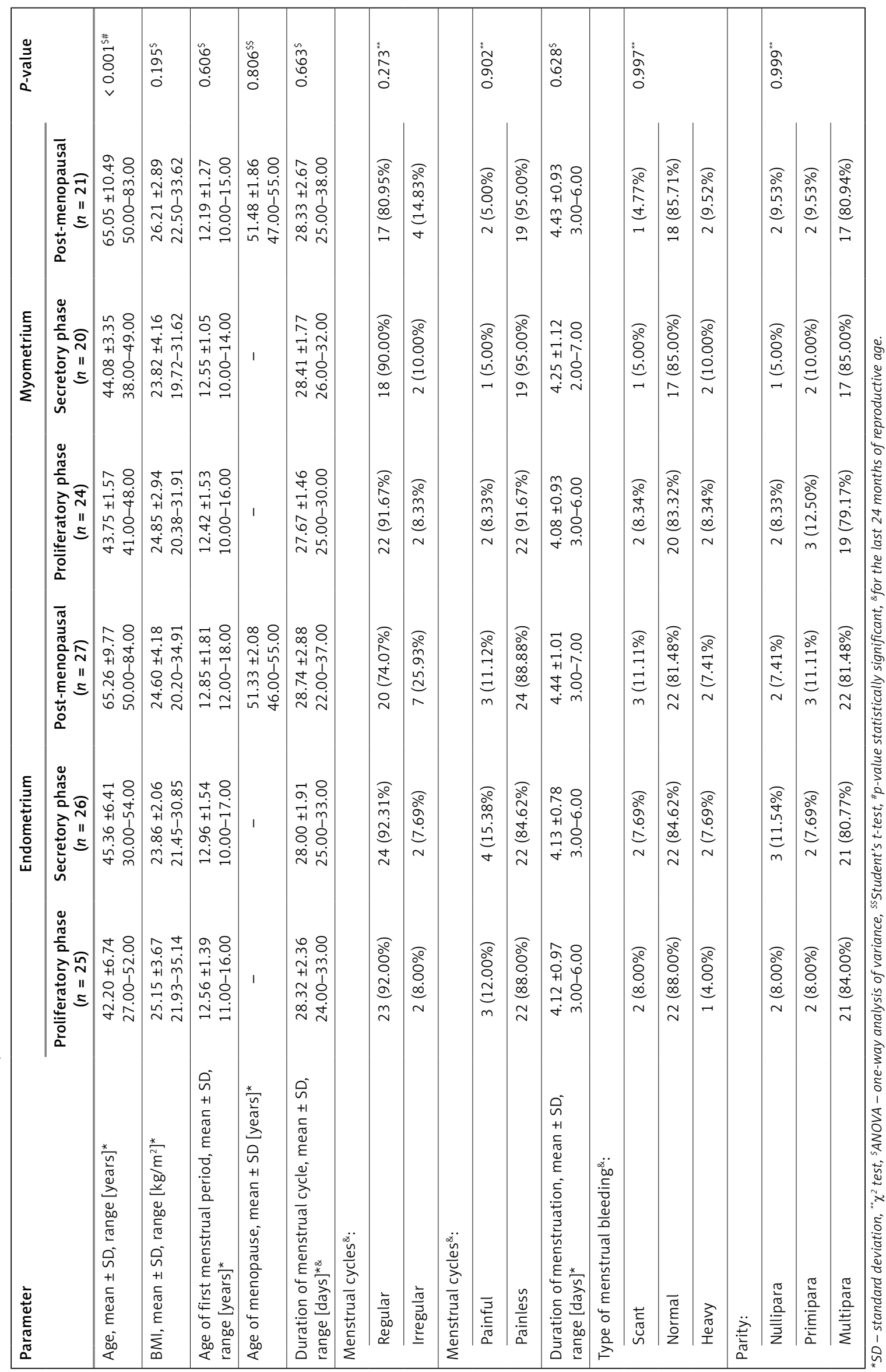



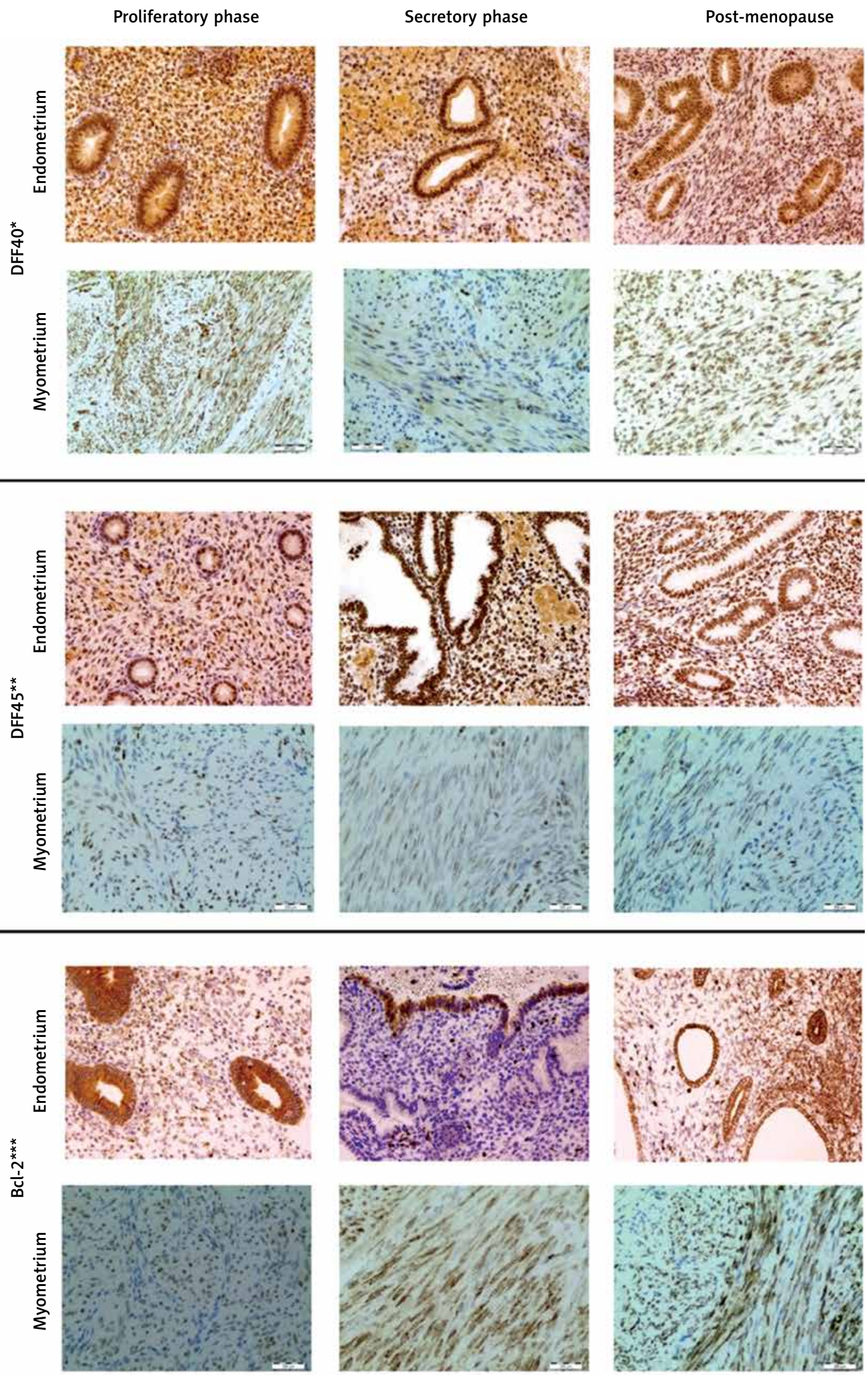

Figure 1. Expression of DFF40*, DFF45 $5^{\star \star}$ and $\mathrm{Bcl}-2^{\star \star \star}(200 \times)$ in normal human endometrium and myometrium ${ }^{*} D N A$ fragmentation factor $40,{ }^{*}$ DNA fragmentation factor 45 , ${ }^{* * *} B$-cell lymphoma 2. 

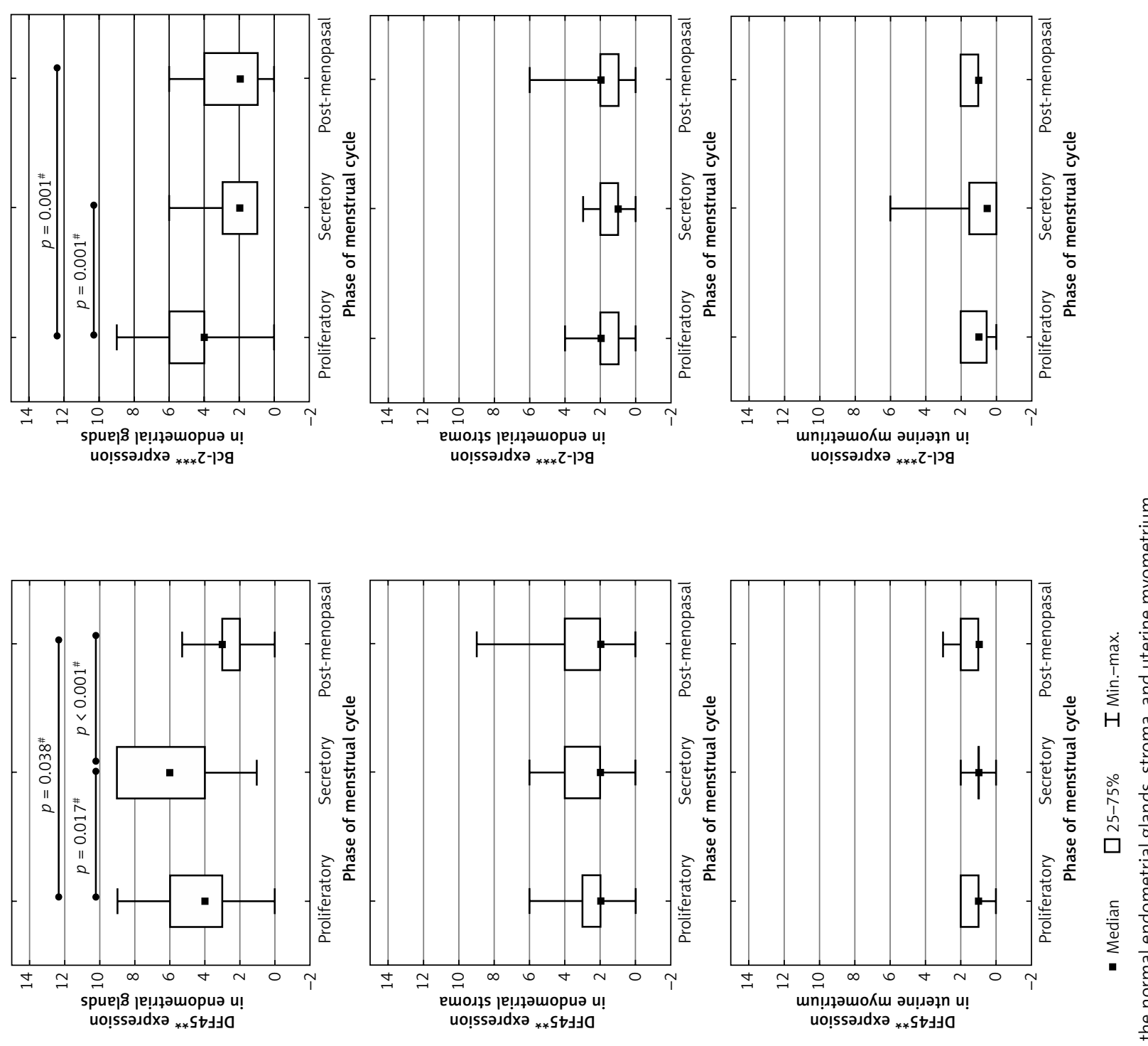

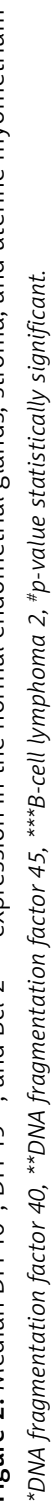

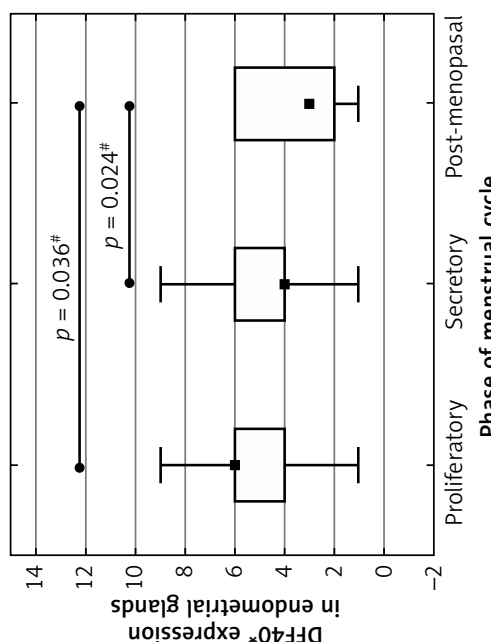

uo!ssəגdxə $* 0 t y=0$

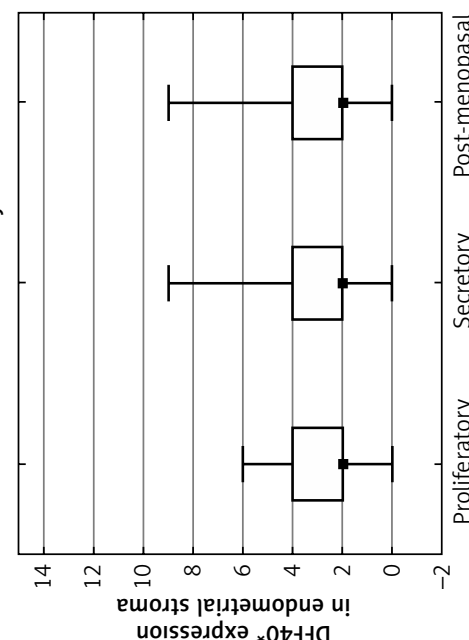

uo!̣səxdxə *0tty

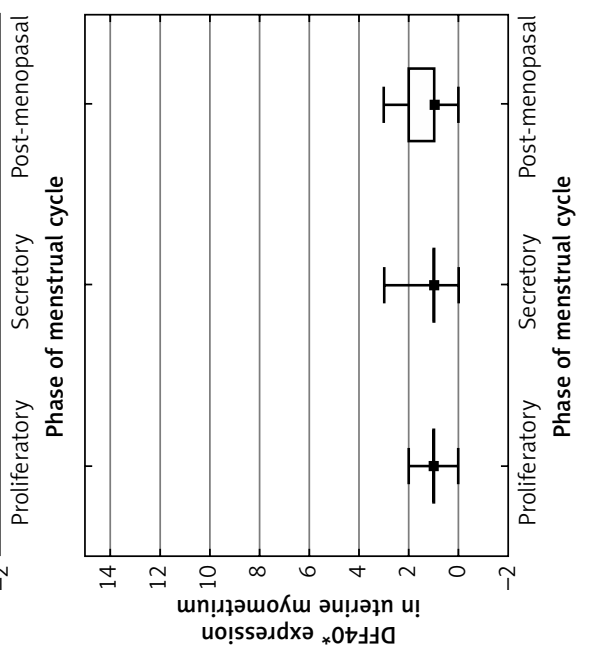


Table II. Immunoexpression of DFF40; DFF45 and Bcl-2 in normal human endometrium and normal human uterine myometrium in different phases of menstrual cycle and postmenopausally

\begin{tabular}{|c|c|c|c|c|c|c|c|c|c|}
\hline \multirow[t]{2}{*}{ Variable } & \multicolumn{6}{|c|}{ Menstrual cycle phase } & \multicolumn{3}{|c|}{ Post-menopausal } \\
\hline & \multicolumn{3}{|c|}{ Proliferatory } & \multicolumn{3}{|c|}{ Secretory } & & & \\
\hline \multicolumn{10}{|c|}{ Median DFF40* expression, (IQR) and min.-max.: } \\
\hline Endometrial glands & & $\begin{array}{l}n \\
\stackrel{0}{0} \\
\dot{0}\end{array}$ & $\begin{array}{r}6.00(2.00) \\
2.00-9.00\end{array}$ & & 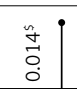 & $\begin{array}{r}6.00(3.00) \\
1.00-9.00\end{array}$ & & i & $\begin{array}{r}4.00(2.00) \\
1.00-6.00\end{array}$ \\
\hline Endometrial stroma & $\begin{array}{l}\vec{O} \\
\dot{0} \\
\dot{0} \\
v\end{array}$ & 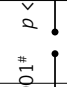 & $\begin{array}{r}3.00(2.00) \\
0.00-9.00\end{array}$ & $\begin{array}{l}\vec{b} \\
0 \\
\dot{0} \\
\text { v }\end{array}$ & 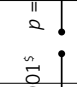 & $\begin{array}{r}3.00(2.00) \\
0.00-9.00\end{array}$ & $\begin{array}{l}\overrightarrow{8} \\
0 \\
\dot{0} \\
\text { v }\end{array}$ & 帮 & $\begin{array}{r}3.00(2.00) \\
1.00-9.00\end{array}$ \\
\hline Uterine myometrium & & $\begin{array}{l}0 \\
0 \\
11 \\
2\end{array}$ & $\begin{array}{r}1.00(1.00) \\
0.00-4.00\end{array}$ & & $\begin{array}{l}0 \\
0 \\
11 \\
0 .\end{array}$ & $\begin{array}{r}1.00(0.50) \\
0.00-3.00\end{array}$ & & $\begin{array}{l}0 \\
0 \\
0 \\
11 \\
2 \\
0\end{array}$ & $\begin{array}{r}2.00(1.00) \\
0.00-3.00\end{array}$ \\
\hline \multicolumn{10}{|c|}{ Median DFF45 ${ }^{\star *}$ expression (IQR) and min.-max.: } \\
\hline Endometrial glands & & $\begin{array}{l}0 \\
\infty \\
\infty \\
0 \\
0 \\
0 \\
0\end{array}$ & $\begin{array}{r}3.00(2.00) \\
0.00-6.00\end{array}$ & & $\begin{array}{l}2 \\
\dot{0} \\
0 \\
0\end{array}$ & $\begin{array}{r}4.00(2.00) \\
2.00-9.00\end{array}$ & & 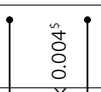 & $\begin{array}{r}2.00(2.00) \\
0.00-6.00\end{array}$ \\
\hline Endometrial stroma & & 2 & $\begin{array}{r}2.00(2.00) \\
0.00-4.00\end{array}$ & $\begin{array}{l}\vec{\sigma} \\
0 \\
0 \\
v \\
\end{array}$ & $\begin{array}{l}2 \\
2 ! \\
* \\
0\end{array}$ & $\begin{array}{r}2.00(2.00) \\
0.00-6.00\end{array}$ & $\begin{array}{l}\overrightarrow{\widetilde{O}} \\
0 \\
0 \\
\text { II }\end{array}$ & e. & $\begin{array}{r}1.00(1.00) \\
0.00-2.00\end{array}$ \\
\hline Uterine myometrium & & & $\begin{array}{r}1.00(1.00) \\
0.00-3.00\end{array}$ & & $\begin{array}{l}0 \\
0 \\
11 \\
0 .\end{array}$ & $\begin{array}{r}1.00(0.00) \\
0.00-2.00\end{array}$ & & & $\begin{array}{r}1.00(0.00) \\
0.00-2.00\end{array}$ \\
\hline \multicolumn{10}{|c|}{ Median Bcl-2*** expression, (IQR) and min.-max.: } \\
\hline Endometrial glands & & $\begin{array}{l}n \\
\overline{8} \\
0 \\
0\end{array}$ & $\begin{array}{r}4.00(0.00) \\
2.00-9.00\end{array}$ & & & $\begin{array}{r}1.00(2.00) \\
0.00-6.00\end{array}$ & & & $\begin{array}{r}2.00(3.00) \\
0.00-6.00\end{array}$ \\
\hline Endometrial stroma & $\begin{array}{l}\overrightarrow{8} \\
\dot{0} \\
\text { v } \\
0\end{array}$ & $\begin{array}{l}2 \\
2 ! \\
* \\
0 \\
0\end{array}$ & $\begin{array}{r}2.00(1.00) \\
0.00-4.00\end{array}$ & & & $\begin{array}{r}1.00(1.00) \\
0.00-2.00\end{array}$ & & & $\begin{array}{r}2.00(1.00) \\
0.00-6.00\end{array}$ \\
\hline Uterine myometrium & & $\begin{array}{l}0 \\
0 \\
0 \\
2 \\
2\end{array}$ & $\begin{array}{r}1.00(1.50) \\
0.00-4.00\end{array}$ & & & $\begin{array}{r}0.50(1.50) \\
0.00-6.00\end{array}$ & & & $\begin{array}{r}1.00(1.00) \\
1.00-4.00\end{array}$ \\
\hline
\end{tabular}

${ }^{\star}$ DNA fragmentation factor $40,{ }^{* *} D N A$ fragmentation factor $45,{ }^{* * *} B$-cell lymphoma $2,{ }^{*}$ Mann-Whitney U-test, ${ }^{\text {spaired }}$ Wilcoxon test.

\section{Validation of the intra-rater reliability for immunohistochemistry scoring}

An almost perfect intra-rater agreement was confirmed with regard to the immunoscoring of DFF40, DFF45, and Bcl-2 expression. The following values were noted:

A) The intra-rater agreement of the first investigator (i.e., investigator 1 vs. 1) for the assessment of DFF40 is as follows: $\kappa=1.0(p<0.001)$; DFF45: $\kappa=0.95(p<0.001)$; and Bcl-2: $\kappa=0.96(p<$ 0.001).

B) The intra-rater agreement of the second investigator (i.e., investigator 2 vs. 2) for the assessment of DFF40 is as follows: $\kappa=1.0(p<0.001)$; DFF45: $\kappa=1.0(p<0.001)$; and Bcl-2: $\kappa=0.96$ $(p<0.001)$.

\section{Discussion}

To the best of our knowledge, this study is the first comprehensive report of the DFF40, DFF45, and $\mathrm{Bcl}-2$ expression in the human uterus under physiological conditions. Our results provide evidence that menstrual cycle-dependent changes in the expression of DFF40, DFF45, and Bcl-2 are present predominantly in the endometrial glandular epithelium. These results are consistent with our previous findings that show changes in DFF45 expression in the human endometrium with respect to the phases of the menstrual cycle $[3,4,8]$.

DFF40 is a major apoptotic nuclease responsible for the final DNA fragmentation in apoptosis, and its high and comparable expression was observed in the endometrial glandular epithelium during both the proliferative and secretory phases of the menstrual cycle. Therefore, we assume that the tissues that show high DFF40 expression are potentially susceptible to apoptosis. According to our results, the endometrial glandular epithelium showed higher potential receptivity to apoptosis than the endometrial stroma and uterine myometrium. These findings are consistent with those of Matsumoto et al., who observed that apoptosis most frequently appeared in the epithelial endometrium [21]. However, it must be noted that DFF40 alone is not sufficient to execute DNA fragmentation, because when DFF40 is in the nucleus, it remains bound to DFF45, which inhibits the activity of DFF40. Conversely, DFF45 plays the dual role of DFF40 inhibitor and chaperone. The expression of DFF40 in the absence of co-expressed DFF45 results in the generation of inactive DFF40 aggregates [22, 23]. In our study, the endometrial glandular epithelium showed the highest DFF45 expression during the secretory phase of the menstrual cycle compared with that 
during the proliferative phase and postmenopausally. Therefore, we assume that increased DFF45 expression in the endometrial glandular epithelium during the secretory phase of the menstrual cycle makes it potentially susceptible to apoptosis. Regression analysis proved that DFF40 and DFF45 expression was independent from most of the clinical features in both the glandular and stromal layer of the endometrium and confirmed that the menopausal status was the only characteristic significantly influencing DFF40 expression in the endometrial glands. Similarly, DFF45 endometrial glandular expression was dependent only on the menstrual cycle phase and menopausal status. In contrast, the endometrium uterine myometrium showed independent DFF40 and DFF45 expression from all the analyzed clinical features.

The current study also confirmed the significant decrease in $\mathrm{Bcl}-2$ expression that occurs predominantly during the secretory phase of the menstrual cycle compared with that during the proliferative phase in the endometrial glandular epithelium. $\mathrm{Bcl}-2$ glandular endometrial expression was dependent on the menstrual cycle phase but no other clinical characteristics. These results are consistent with those of Otsuki et al., who reported that glandular endometrial cells express $\mathrm{Bcl}-2$ during the proliferative phase of the menstrual cycle through the early secretory phases (but not during the late secretory phase) [24]. Furthermore, these authors found that the disappearance of $\mathrm{BCl}-2$ expression was correlated with the appearance of apoptosis. In addition, Li et al. [25] postulated that the binding of c-Jun to estrogen receptor $\alpha$ regulates the proliferative phase-specific expression of the $\mathrm{Bcl}-2$ gene in glandular endometrial cells. The high $\mathrm{Bcl}-2$ expression and low DFF45 expression that occurs during the proliferative phase of the menstrual cycle may prevent apoptosis, even when the level of DFF40 remains consistently high, while the decreased $\mathrm{Bcl}-2$ and increased DFF45 expression that occurs during the secretory phase of the menstrual cycle may enhance apoptosis.

Although our outcomes are consistent with the data obtained by other researchers, the present study has limitations. First, immunostaining was employed as the only study technique, and this semi-quantitative method does not allow us to directly compare the DFF40 and DFF45 levels with each other. However, the roles that the DFF40/ DFF45 complex and $\mathrm{Bcl}-2$ play in apoptosis have already been explained, and this goal was not the aim of our study. In addition, we realize that an immunostaining analysis is subjective. Therefore, two experienced pathologists evaluated each sample, and a discrepancy occurred only in $2.74 \%$ of cases. These differences were reevaluated thereafter to achieve a final consensus. This methodology is widely accepted and employed by many other studies regarding the expression of DFF45 and Bcl-2 [3, 4, 8-12, 16]. As complete inter-rater agreement was achieved by creating a consensus in ambiguous cases, the intra-rater disparity was the only potential bias in our sample assessment. This disparity showed an almost perfect correlation; therefore, intra-rater bias can be safely excluded. Immunostaining was selected as an investigation method for the following reasons: first, this approach allows our findings to be compared with previously published results; second, because immunochemistry is a widely used method in pathomorphological laboratories, this technique can be easily implemented and performed if our assessments of DFF40, DFF45, or Bcl-2 reach clinical applications. We also considered using an automatic assessment of digitalized whole slide images instead of pathologist-performed immunoscoring. An exact discernment between the endometrial glandular epithelium and stroma was required, which could be best provided by pathologists. Moreover, we did not find any description of this method regarding the immunoscoring of the DFF40/DFF45 complex. Thus, to avoid the potential bias caused by implementation of a new methodology, we decided to abandon this method.

The reliable histopathological classification of the specimens remains the core strength of our study. The paraffin-embedded slides were properly stored and well prepared, which allowed for repeated immunostaining to be achieved for each sample, providing a brief period of retrospective analysis. Suitable selection of cases, based on their medical history, hysteroscopic and histopathology results, allowed us to avoid bias related to underlying and unidentified endometrial and myometrial pathology.

In conclusion, the current study provides important evidence regarding menstrual cycle-dependent changes in the expression of DFF40/ DFF45 and Bcl-2 in the normal human endometrium, especially in the glandular layer, and shows that their levels are stable in the normal uterine myometrium. This comprehensive evaluation provides a better understanding of other findings concerning DFF45 and $\mathrm{Bcl}-2$ expression in female genital tract pathologies, including malignancies.

\section{Acknowledgments}

The authors thank Dr. H. Molak-Olczak, who independently and blindly analyzed the immunoexpression of the investigated proteins as a second specialist in histopathology.

\section{Conflict of interest}

The authors declare no conflict of interest. 


\section{References}

1. Harada T, Kaponis A, Iwabe T, et al. Apoptosis in human endometrium and endometriosis. Hum Reprod Update 2004; 10: 29-38.

2. Torrijos MC, de Merlo GG, Mirasol EG, García MT, Parra CÁ, Goy El. Endometrial study in patients with postmenopausal metrorrhagia. Arch Med Sci 2016; 12: 597-602.

3. Banas T, Basta P, Knafel A, et al. DFF45 expression in human endometrium is associated with menstrual cycle phases and decreases after menopause. Gynecol Obstet Invest 2012; 73: 177-82.

4. Popiela TJ, Wicherek L, Radwan M, et al. The differences in RCAS1 and DFF45 endometrial expression between late proliferative, early secretory, and mid-secretory cycle phases. Folia Histochem Cytobiol 2007; 45 Suppl 1: S157-62.

5. Sakahira H, Enari M, Nagata S. Cleavage of CAD inhibitor in CAD activation and DNA degradation during apoptosis. Nature 1998; 391: 96-9.

6. Liu X, Zou H, Widlak P, Garrard W, Wang X. Activation of the apoptotic endonuclease DFF40 (caspase-activated DNase or nuclease). Oligomerization and direct interaction with histone H1. J Biol Chem 1999; 274: 13836-40.

7. Widlak P, Garrard WT. Roles of the major apoptotic nuclease-DNA fragmentation factor in biology and disease. Cell Mol Life Sci 2009; 66: 263-74.

8. Banas T, Skotniczny K, Basta A. DFF45 expression in ovarian endometriomas. EJOGRB 2009; 146: 87-91.

9. Brustmann H. Poly(ADP-ribose) polymerase (PARP) and DNA-fragmentation factor (DFF45): expression and correlation in normal, hyperplastic and neoplastic endometrial tissues. Pathol Res Pract 2007; 203: 65-72.

10. Brustmann H. DNA fragmentation factor (DFF45): expression and prognostic value in serous ovarian cancer. Pathol Res Pract 2006; 202: 713-20.

11. Charrier L, Jarry A, Toquet C, et al. Growth phase-dependent expression of ICAD-L/DFF45 modulates the pattern of apoptosis in human colonic cancer cells. Cancer Res 2002; 62: 2169-74.

12. Konishi S, Ishiguro H, Shibata Y, et al. Decreased expression of DFF45/ICAD is correlated with a poor prognosis in patients with esophageal carcinoma. Cancer 2002; 95: 2473-8.

13. Sánchez-Osuna M, Martínez-Escardó L, GranadosColomina C, et al. An intrinsic DFF40/CAD endonuclease deficiency impairs oligonucleosomal DNA hydrolysis during caspase-dependent cell death: a common trait in human glioblastoma cells. Neuro Oncol 2016; 18: 950-61.

14. Pinheiro A, Antunes A Jr, Andrade L, De Brot L, Pinto-Neto $A M$, Costa-Paiva L. Expression of hormone receptors, $\mathrm{Bcl}-2$, Cox-2 and Ki67 in benign endometrial polyps and their association with obesity. Mol Med Rep 2014; 9: 2335-41.

15. Bozkurt KK, Yalçın Y, Erdemoğlu E, et al. The role of immunohistochemical adrenomedullin and $\mathrm{Bcl}-2$ expression in development of type-1 endometrial adenocarcinoma: adrenomedullin expression in endometrium. Pathol Res Pract 2016; 212: 450-5.

16. Korkmaz D, Bastu E, Dural O, Yasa C, Yavuz E, Buyru F. Apoptosis through regulation of $\mathrm{BCl}-2, \mathrm{Bax}$ and $\mathrm{Mcl}-1$ expressions in endometriotic cyst lesions and the endometrium of women with moderate to severe endometriosis. J Obstet Gynaecol 2013; 33: 725-8.

17. Nikolic I, Andjelkovic M, Zaric M, et al. Induction of mitochondrial apoptotic pathway by raloxifene and estrogen in human endometrial stromal ThESC cell line. Arch Med Sci 2017; 13: 293-301.

18. Eder P, Łykowska-Szuber L, Krela-Kaźmierczak I, et al. Disturbances in apoptosis of lamina propria lymphocytes in Crohn's disease. Arch Med Sci 2015; 11: 1279-85.

19. Newmeyer DD, Bossy-Wetzel E, Kluck RM, Wolf BB, Beere HM, Green DR. Bcl-x(L) does not inhibit the functuion of Apaf-1. Cell Death Diff 2000; 7: 402-7.

20. Kottner J, Audige L, Brorson S, et al. Guidelines for Reporting Reliability and Agreement Studies (GRRAS) were proposed. J Clin Epidemiol 2011; 64: 96-106.

21. Matsumoto Y, Iwasaka T, Yamasaki F, Sugimori H. Apoptosis and $\mathrm{Ki}-67$ expression in adenomyotic lesions and in the corresponding eutopic endometrium. Obstet Gynecol 1999; 94: 71-7.

22. Liu X, Zou H, Slaughter C, Wang X. DFF, a heterodimeric protein that functions downstream of caspase-3 to trigger DNA fragmentation during apoptosis. Cell 1997; 89: 175-84

23. Liu X, Li P, Widłak P, Zou H, Luo X, Garrard WT, Wang X. DFF40 induces DNA fragmentation and chromatin condensation during apoptosis. Proc Natl Acad Sci USA 1998; 95: 8461-6.

24. Otsuki Y, Misaki O, Sugimoto O, Ito Y, Tsujimoto Y, Akao Y. Cyclic bcl-2 gene expression in human uterine endometrium during menstrual cycle. Lancet 1994; 344: 28-9.

25. Li ZL, Ueki K, Kumagai K, Araki R, Otsuki Y. Regulation of bcl-2 transcription by estrogen receptor-alpha and c-Jun in human endometrium. Med Mol Morphol 2014; 47: 43-53. 